\title{
The association of anxiety and depression with musculoskeletal disorders among military personnel in 2016
}

\author{
Ghanbary Sartang A, MSc ${ }^{1}$, Ashnagar M, $\mathrm{MSc}^{2 *}$, Habibi E, $\mathrm{PhD}^{3}$, Sadeghi S, MSc ${ }^{4}$ \\ 1- MSc Occupational Health, School of Health, Isfahan University of Medical Sciences, Isfahan, Iran. 2- MSc Industrial \\ Psychology, Bandar Abbas, Iran. 3- Professor, Dept. of Occupational Health Engineering, School of Health, Isfahan \\ University of Medical Sciences, Isfahan, Iran. 4- MSc of Industrial Engineering, Dept. of Industrial Engineering, Islamic \\ Azad University Ilam, Ilam, Iran
}

\begin{abstract}
Received: December 2016, Accepted: February 2017

Background: Musculoskeletal pain accompanied by psychological problems leads to reduced health efficiency. On the other hand, work-related anxiety and depression is another important factor that could result in reduced productivity in organizations and physical and mental problems in personnel. Musculoskeletal disorders (MSDs) are the most common occupational health problems that have high costs and lead to productivity loss in military personnel. The purpose of this study was to evaluate the association of anxiety and depression with MSDs among military personnel in 2016.

Materials and Methods: This descriptive-analytic and cross-sectional study was conducted on 70 military personnel in July 2016 through convenience sampling method. The data collection tools used were the Cornell Musculoskeletal Discomfort Questionnaire (CMDQ) and Goldberg Anxiety and Depression Scale. Data analysis was conducted using descriptive statistics, Pearson correlation test, and ANOVA in SPSS software.

Results: The highest prevalence of MSDs was observed in the back (48\%), thoracic spine (41\%), and shoulder (37\%). The Goldberg Anxiety and Depression Scale scores showed that anxiety and depression were at a medium level. The Pearson correlation test showed a significant correlation between anxiety $(\mathrm{P}=0.01)(\mathrm{r}=0.79)$ and depression $(\mathrm{P}=0.01)(\mathrm{r}=0.76)$, and MSDs; with increase in anxiety and depression, MSDs also increased.

Conclusions: The results of this study showed that anxiety and depression have a direct relationship with MSDs. Therefore, interventions must be carried out for MSDs prevention such as reducing physical and psychological job demands, and reducing anxiety and depression level among military personnel.
\end{abstract}

Keywords: Anxiety, Depression, Musculoskeletal Disorder, Military Personnel

\section{Introduction}

Pain in the workplace is any chronic ailment that occurs as a result of work or occupational activity. Moreover, pain in the workplace is typically identified through its higher prevalence in a given body of workers than in the general population, or in other worker populations. Musculoskeletal conditions and mental disorders cause few deaths but much pain, disability and distress in military personnel (1). Anxiety and depression are an inevitable part of our modern life and may make various diseases (2). MSDs are the most common health problem associated with work in Europe, affecting millions of workers. In addition, 25\% of European workers complain of back pain and $23 \%$ of muscle aches (3). Work-related MSDs are groups of syndromes characterized by symptoms of soft tissue pain, par aesthesia, stiffness, swelling, weakness, discomfort, and loss of function that can be caused or aggravated by work-related exposure (4). MSDs are the main cause of sickness absence in Western European countries. Factors associated with MSDs include demographic characteristics, such as

* Corresponding author: Mehdi Ashnagar, MSc Industrial Psychology, Bandar Abbas, Iran. E-mail: m.ashna63@yahoo.com 
age and sex, occupational risk factors, and non-work related exposures (5). In a study on injury-related musculoskeletal conditions among air force, army, and navy soldiers, and marines, Hauret et al. concluded that of the 743,547 injuries, $82 \%$ were classified as inflammation/pain/over-use (6). Injuries involving the vertebral column and lower extremity accounted for nearly equal proportions of all injuries (40 and 39\%, respectively) while upper extremity injuries comprised $14 \%$ of total injuries (6). Mental and behavioral disorders, low back pain, and MSDs are among the top 20 most common diseases. Anxiety and depressive disorders are among the most prevalent psychiatric disorders. They comprise a wide range of different disorders. Most anxiety disorders first appear during childhood and adolescence (7). If anxiety and depression exceed their threshold values, the result is reduced efficiency through reduced attention, sleep disruption, increased risk of disease, irreparable mistakes, and even harassment and violence at work, work absences, burnout, and reduced work performance (8). general health was presented for the first time by Goldberg in 1972 and is one of the anxiety and depression assessment methods (9). Anxiety is an emotion characterized by feelings of tension, worried thoughts, and physical changes like increased blood pressure. People with anxiety disorders usually have recurring intrusive thoughts or concerns. Anxiety reaction to danger is uncontrollable. Anxiety is accompanied with one or more physical sensations such as shortness of breath, palpitations, sweating, headache, and restlessness. Anxiety is a sign of many psychological disorders (10).

Depression is a mood characterized by feelings of inadequacy and incompetence, poor perceptions of oneself, and associated physical, behavioral, and cognitive symptoms. Some of its symptoms include disinterest, lack of joy, nostalgia, inactivity, and sense of disturbance. Depression is a state of low mood and aversion to activity or apathy that can affect a person's thoughts, behavior, feelings, and sense of well-being. People with a depressed mood can feel sad, anxious, empty, hopeless, helpless, worthless, guilty, irritable, and angry (11). Military personnel are faced with multiple stressors of great importance such as depression, anxiety, and MSDs. There is no evidence of the association of anxiety and depression with MSDs among military personnel. Military personnel are exposed to physical strain as an inherent part of their job, and as a consequence, are at risk of morbidity in terms of injuries and MSDs in general. The purpose of this study was to evaluate the association of anxiety and depression with MSDs among military personnel in 2016.

\section{Material and Methods}

The present cross-sectional and correlational study was conducted on 70 military personnel. Written consent forms were obtained from all those who accepted to participate in the study after receiving details about the study methods and objectives. The subjects were selected through simple random sampling. The inclusion criteria included lack of history of hypertension or other medical problems (such as musculoskeletal ache), and a minimum of 6 months of work experience. The exclusion criteria consisted of pain or discomfort in various body organs, and unwillingness to participate in the study. Data were collected using the Goldberg Anxiety and Depression Scale and Cornell Musculoskeletal Discomfort Questionnaire (CMDQ). In the next stage, after explaining the research objectives for the participants, assuring them of confidentiality, and obtaining written consent forms, questionnaires were simultaneously distributed among the participants. The Goldberg Anxiety and Depression Scale were designed by Goldberg et al. in 1979. The demographic variables of age, level of education, height, weight, and work experience were recorded, and then, the CMDQ and Goldberg Anxiety and Depression Scale were completed. The CMDQ is through observation method that can 
identify ergonomic risk factors and has good reliability for the assessment of MSDs. The CMDQ is a self-report questionnaire that qualitatively assesses MSDs of the neck, shoulders, thoracic spine, back, forearm, wrist, hand, thigh, buttock, knee, and foot. The validity and reliability of the CMDQ have been approved in the study by Affifehzadeh et al. and the Cronbach's alpha for this scale was reported as 0.98 (12). The Goldberg Anxiety Scale include 7 questions and Goldberg Depression Scale include 7 questions that have be taken from the General Health Questionnaire. The items of this questionnaire were scored on a 4-point Likert scale $(0=$ never, $1=$ sometimes, $2=$ often, and $3=$ always). The validity and reliability of this questionnaire were confirmed in the study by Taghavi et al. and Cronbach's alpha of the Anxiety and Depression Scales was, respectively, 0.84 and 0.88 . The minimum and maximum score for this scale is 0 and 21 , respectively. The total score of 0-6, 7-11, 1216 , and 17-21 represented the least anxiety or depression, mild anxiety or depression, moderate anxiety or depression, and severe anxiety or depression, respectively (13). Finally, data analysis was conducted using descriptive statistics, Pearson correlation test, and ANOVA in SPSS software (version 20, SPSS Inc., Chicago, IL, USA). All P values of less than 0.05 were considered significant. The normality of data was verified using the Kolmogorov-Smirnov test.

\section{Results}

In this study, 70 military men with the average age of $34.08 \pm 3.81$ years participated. The maximum and minimum ages of participants in the study were 49 and 25 years, respectively. The average work experience of participants in this study was $14.2 \pm 5.35$ years. The maximum and minimum work experience of participants in the study was 28 years and 1 year, respectively. In terms of marital status, $50(71.4 \%)$ participants were married and $20(29.6 \%)$ were single. The number of participants based on level of education is presented in table 1 .

Table 1: The number of participants by level of education (70 people)

\begin{tabular}{lll}
\hline Variable & Number & Percentage \\
\hline Diploma & 6 & 8.5 \\
\hline Associate degree & 25 & 35.71 \\
\hline Bachelor's degree & 33 & 47.29 \\
\hline Master's degree & 6 & 8.5 \\
\hline
\end{tabular}

Demographic characteristics of height, weight, age, and work experience are presented in table 2. Average score of the Anxiety Scale was $13.82 \pm 2.14$ and the average score of the Depression Scale was $12.34 \pm 1.84$. Scores obtained from the questionnaires showed that anxiety and depression are at a medium level. In figure 1 , mean \pm SD scores of anxiety and depression have been shown in a pie chart and the average score of anxiety is higher than depression.

Table 2: Demographic characteristics of weight, age, height, and work experience

\begin{tabular}{lll}
\hline Variable & Mean \pm SD & Maximum-Minimum \\
\hline Height $(\mathrm{cm})$ & $180.2 \pm 5.2$ & $189-166$ \\
\hline Weight $(\mathrm{kg})$ & $78.35 \pm 8.6$ & $97-64$ \\
\hline Age & $34.08 \pm 3.81$ & $49-25$ \\
\hline Work experience & $14.2 \pm 5.35$ & $28-1$ \\
\hline
\end{tabular}




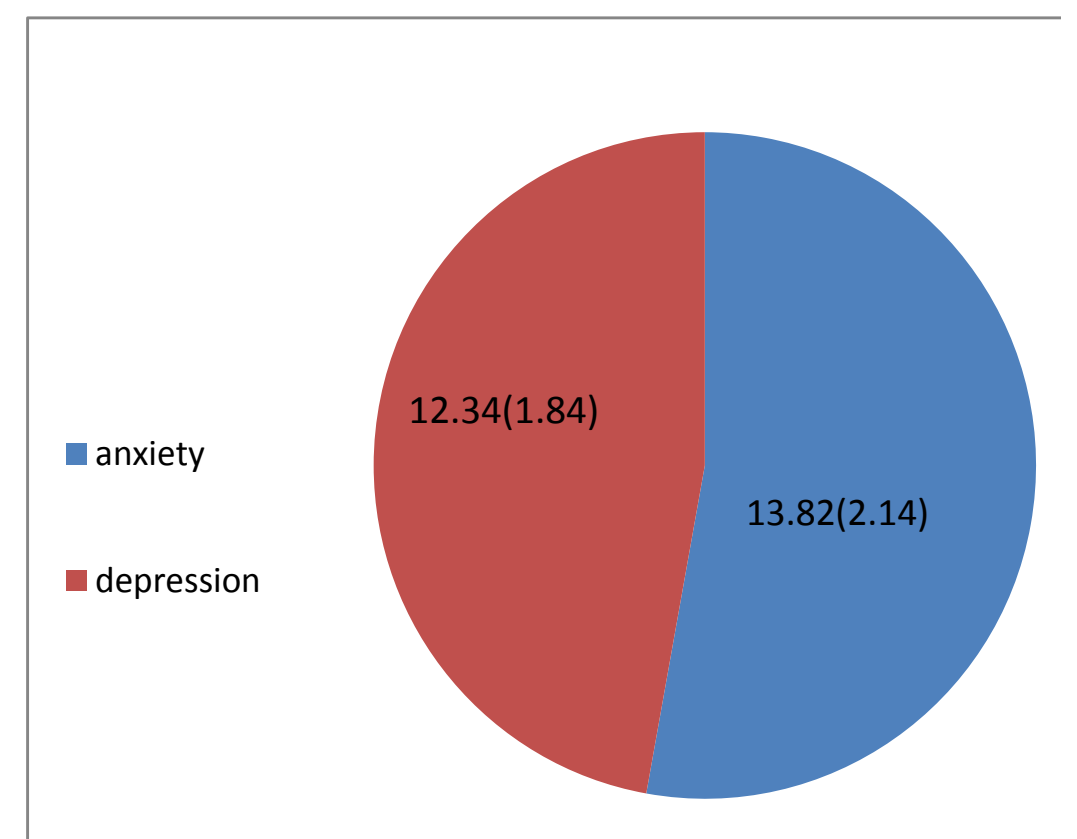

Figure 1: Mean \pm SD scores of anxiety and depression

Using the CMDQ, the prevalence of MSDs in different organs was determined during the previous 12 months and the most prevalent MSDs were, respectively, in the back (48\%), thoracic spine (41\%), and shoulder (37\%). The percentage of MSDs in different organs based on the CMDQ during the previous 12 months is presented in table 3 .

Table 3: Musculoskeletal disorders in different organs during the previous 12 months $(\mathrm{n}=70$ people)

\begin{tabular}{lll}
\hline Variable & Number & Percentage \\
\hline Neck & 25 & 35 \\
\hline Shoulder & 26 & 37 \\
\hline Arm & 14 & 2 \\
\hline Thoracic spine & 29 & 41 \\
\hline Forearm & 10 & 14 \\
\hline Buttock & 9 & 12 \\
\hline Wrist & 19 & 27 \\
\hline Back & 34 & 48 \\
\hline Thigh & 12 & 17 \\
\hline Knee & 21 & 30 \\
\hline Legs & 19 & 27 \\
\hline
\end{tabular}

To investigate the association of anxiety and depression with MSDs, the Pearson correlation test was used. Pearson correlation test showed a significant direct association between anxiety $(\mathrm{P}=0.01)(\mathrm{r}=0.79)$ and depression ( $\mathrm{P}$ $=0.01)(\mathrm{r}=0.76)$ scores and MSDs; with increase in anxiety and depression, MSDs also increased. Kolmogorov-Smirnov test confirmed the normal distribution of variables, and ANOVA showed that with increasing age $(\mathrm{P}=0.04)$ and work experience $(\mathrm{P}=0.03)$,
MSDs also increased. Furthermore, age ( $\mathrm{P}=$ 0.02) $(\mathrm{P}=0.03)$ and work experience $(\mathrm{P}=$ 0.03) $(\mathrm{P}=0.02)$ had significant correlations with anxiety and depression, respectively. Anxiety and depression increased with increase in age and work experience.

\section{Discussion}

Among senior military personnel, musculoskeletal conditions are a major factor in medical downgrading, disability, and 
medical discharges from the army. It is wellknown that the prevalence of acute overuse injuries among basic trainees and recruits is high. Depression and anxiety are significant contributors to the global burden of disease and affect individuals in all communities across the world. MSds are the most common occupational health problem reported with high direct costs for diagnosis and treatment and indirect costs of work absenteeism and loss of skilled manpower. In recent years, the role of psychological factors in increasing workplace MSDs has been recognized and psychological factors showed increased risk of musculoskeletal disorders. In the present study, $8.47 \%, 35.86 \%, 47.14 \%$, and $9.53 \%$ of participants had a diploma associate's degree, bachelor's degree, and master's degree, respectively. The results of this study showed the moderate status of anxiety and depression in the study population. Moreover, with increased anxiety and depression, MSDs also increased. Tabatabaei et al. investigated MSDs among emergency medical service personnel and concluded that MSDs increased with increased depression rates (14). This finding was consistent with the findings of this study. The results of the CMDQ showed the highest rate of MSDs, respectively, in the back (48\%), thoracic spine $(41 \%)$, and shoulder $(37 \%)$. Kangarlou et al. studied the prevalence of MSDs in the personnel of army air forces and showed MSDs in the back (51.2\%) and thoracic spine (16.5\%) (15). This finding confirms that of the present study. Aminian et al. concluded that psychological factors and stress are associated with MSDs; with increase in stress and anxiety, MSDs increased, which confirmed the findings of the present study (16). The results showed that MSDs increased with increasing age and work experience. Ghanbari et al. also concluded that MSDs increased as a result of increase in age and work experience (17). Several studies have shown that anxiety and stress play an important role in MSDs and reduce quality of life (QOL) (18). Violante et al. concluded that psychosocial stress and anxiety have an important role in increased depression and MSDs (19). Bolghanabadi et al. evaluated MSDs, occupational fatigue, and occupational mental stress and observed a significant relationship between MSDs, and mental stress and occupational fatigue (20). Shan et al. detected a significantly higher depression score with low back pain compared to neck pain (21). They stated that the reason might be that students are more willing to lessen neck pain, especially those who have more psychiatric symptoms (21). On the association between psychological factors and the incidence of musculoskeletal injuries, it can be said that dissatisfaction with and stress from work are among the factors that are closely related to MSDs (22). Glad et al. in a study on the occurrence and severity of MSDs in Swedish military personnel during peacekeeping operations in Afghanistan during 6 months reported 26\% MSDs in lumbar spine, $26 \%$ in shoulders, and $22 \%$ in lower extremities (23). The limitation of this study was the lack of opportunity to utilize a larger sample size. Moreover, longitudinal studies are needed in this regard to assess directionality and causality.

\section{Conclusion}

The results obtained in this study indicate the presence of a direct relationship between anxiety and depression, and MSDs. The prevalence of MSDs and increased anxiety and depression play an important role in reducing work efficiency, job satisfaction, and increasing employee disability, and thus, require preventive measures. Consequently, based on the results of this study, Actions such as avoiding prolonged sitting, design appropriate workstation and a comprehensive program to reduce psychological stress and mental health can be done.

\section{Acknowledgements}

The present study was performed in and received financial support from a military center. We would like to thank all those who 
cooperated in this research project, we particularly appreciate the cooperation of $\mathrm{Mr}$ Mahdavi.

Conflict of interest: None declared.

\section{References}

1. Boubaker K, Colantoni A, Allegrini E, Longo L, Di Giacinto S, Monarca D, et al. A model for musculoskeletal disorder-related fatigue in upper limb manipulation during industrial vegetables sorting. Int $\mathbf{J}$ Ind Ergon 2014; 44(4):601-5.

2. Lath SK. A study of the occupational stress among teachers. International Journal of Education Administration: Challenges and Prospects 2010; 2(2):421-32 .

3. Murray CJ, Vost T, Lozano R, Naghavi M, Flaxman AD, Michud C, et al. Disabilityadjusted life years (DALYs) for 291 diseases and injuries in 21 regions, 1990-2010: a systematic analysis for the Global Burden of Disease Study 2010. Lancet 2012; 380(9859):2197-223.

4. Chubineh A. Posture analysis methods in occupational ergonomics. $2^{\text {nd }}$ ed. Hamedan: Fanavaran publication; 2009. P.2-50.

5. Shahrivar Z, Tehrani-Doost M, Pakbaz B, Rezaie A, Ahmadi F. Normative data and psychometric properties of the parent and teacher versions of the strengths and difficulties questionnaire (SDQ) in an Iranian community sample. J Res Med Sci 2009; 14(2):69-77.

6. Hauret KG, Jones BH, Bullock SH, CanhamChervak M, Canada S. Musculoskeletal injuries: description of an under-recognized injury problem among military personnel. Am J Prev Med 2010; 38(1 Suppl):S61-70.

7. Naghavi M, Abolhassani F, Pourmalek F, Lakeh M, Jafari N, Vaseghi S, et al. The burden of disease and injury in Iran 2003. Popul Health Metr 2009; 7(1):9. doi: 10.1186/1478-7954-7-9.

8. Khalilzadeh R, Yavarian R, Khalkhali HR. The relationship of job stress, depression and anxiety of nursing staff of Urmia University of Medical Sciences. Journal of Nursing and Midwifery Urmia University of Medical Sciences 2005; 3(1):10-7.

9. Goldberg DP, Hillier VF. A scaled version of the General Health Questionnaire. Psychol Med 1979; 9(1):139-45.

10. Kaviani H, Mousavi AS. Psychometric properties of the persian version of Beck
Anxiety Inventory (BAI). Tehran University Medical Journal 2008; 66(2):136-40.

11. Rajabi Gh. Psychometric properties of Beck Depression Inventory short form items (BDI13). Journal of Iranian Psychologists 2005; 1(4):291-8.

12. Afifehzadeh-Kashani $\mathrm{H}$, Choobineh A, Bakand Sh, Gohari MR, Abbastabar H, Moshtaghi P. Validity and reliability of farsi version of Cornell Musculoskeletal Discomfort Questionnaire (CMDQ). Iran Occupational Health Journal 2011; 7(4):69-75.

13. Taghavi SMR. Validity and reliability of the General Health Questionnaire (GHQ-28) in college students of Shiraz university. Journal of Psychology 2002; 5(4):381-98.

14. Tabatabaei S, Maleki A, Mataji M, Nurian RL, Karimi MA . Investigation of depression, anxiety, and musculoskeletal disorders in emergency staff of hospitals supervised by Shahid Beheshti University of Medical Sciences. Iran Occupational Health Journal 2015; 12(1):55 -65.

15. Kangarlo HR, Malekzadeh Sh, Alizadeh K, Zarei S, Shamshiri B. The Prevalence of musculoskeletal cause of disability retirement among air force personnel during 1993-2004. Annals of Military and Health Sciences Research 2006; 4(2):813-8.

16. Aminian O, Pouryaghoub GH, Shanbeh M. One year study of musculoskeletal disorders and their relation to occupational stress among office workers: a brief report. Tehran University Medical Journal 2012; 70(3):194-9.

17. Ghanbary-Sartang A, Habibi H. Evaluation of musculoskeletal disorders to method Rapid Office Strain Assessment (ROSA) in computers users. Journal of Preventive Medicine 2015; 2(1):47-54.

18. Li Y, Chen KR, Wu CH, Sung FC, et al. Job stress and dissatisfaction in association with non-fatal injuries and the job in a acrosssectional sample of petrochemical workers. Occup Med (Lond) 2001; 51(1):50-5

19. Violante FS, Fiori M, Fiorentini C, Risi A, Garagnani G, Bonfiglioli R, et al. Associations of psychosocial and individual factors with three different categories of back disorder among nursing staff. J Occup Health 2004; 46(2):100-8.

20. Bolghanabadi S, Dehghan H, Pour M. The relationship between musculoskeletal disorders, stress and fatigue in the food industry employees. Journal of Ergonomics 2014; 2(1):54-63.

21. Shan Z, Deng G, Li J, Li Y, Zhang Y, Zhao Q. Correlational analysis of neck/shoulder pain and low back pain with the use of digital 
products, physical activity and psychological status among adolescents in Shanghai. PLos One 2013; 8(10):e78109. doi: 10.1371/journal.pone.0078109

22. Eatough EM, Way JD, Chang $\mathrm{CH}$. Understanding the link between psychosocial work stressors and work-related musculoskeletal complaints. Appl Ergon 2012; 43(3):554-63

23. Glad D, Skillgate E, Holm LW. The occurrence and severity of musculoskeletal disorders in Swedish military personnel during peacekeeping operations in Afghanistan. Eur Spine J 2012; 21(4):739-44. 